\title{
A Review on 3D Image Watermarking and Quality Assessment
}

\author{
Divya $^{1}$, Sreeleja N Unnithan ${ }^{2}$ \\ PG Scholar, ECE Department, NSS College of Engineering, Palakkad, Kerala, India ${ }^{1}$ \\ Associate Professor, ECE Department, NSS College of Engineering, Palakkad, Kerala, India ${ }^{2}$
}

\begin{abstract}
The increasing amount of research on watermarking over the past decade has been largely driven by its important applications in digital copyrights management and protection. Digital watermarking is the act of hiding a message related to a digital signal (i.e. an image, song, video) within the signal itself. It is a concept closely related to steganography, in that they both hide a message inside a digital signal. However, what separates them is their goal. Watermarking tries to hide a message related to the actual content of the digital signal, while in steganography the digital signal has no relation to the message, and it is merely used as a cover to hide its existence. With the emerging of low cost 3D display devices, different kinds of 3D applications and the amount of 3D content are booming up, recently. Effective digital watermarking systems for 3D content protection will play an important role for developing and promoting the 3D entertainment industry. The proposed 3D watermarking technique employs the method of slice extraction from a 3D volume. An obj file is used and the wave front object data is obtained.DWT technique is used to embed the secret binary data into one of the extracted slices. The inverse operation is performed at the receiver using the same secret key used at the transmitter. Finally, the watermark is extracted and the quality measures of the reconstructed image is calculated.
\end{abstract}

Keywords: Digital watermark, obj file, Slice extraction, DWT, MSE, PSNR

\section{INTRODUCTION}

3D images are widely used in medical imaging, virtual reality, computer aided design, video games and many other applications. Recently, different types of 3D applications and the uses of 3D content are growing up, with the emerging of low cost 3D display devices[2]. Due to an increased demand for handling 3D models as digital media in the production of computer animation films and video games, different techniques for watermarking 3D models have received much more attention in recent years especially for the copyright protection [4]. Basically, watermarking of 3D models itself is more involved because such a model is usually represented as a 3D triangular mesh, where the vertices, edges, and faces together constitute an arbitrary topological connectivity except for the condition that every face is a triangle. This form of representation is quite different from traditional media such as images and videos because, for such types of media, regular grid samples are assumed in general[3].

3D image modelling has been recently deployed in multiple applications such as virtual reality, motion pictures, video games and medical imaging. This is because of the pervasive advancement in computer graphics and visualization software and hardware tools. Similarly, 3D image steganography has become an interesting field of research due to its enlarged embedding capacity, compared to 2D images[5]. An OBJ file is a standard 3D image format that can be exported and opened by various 3D image editing programs. It contains a three-dimensional object including 3D coordinates, texture maps, polygonal faces, and other object information.

\section{3D WATERMARKING TECHNIQUES}

The progressive increment in the usage of 3D images in several fields also increases the need of security.An obj file is watermarked here.There are many other techniques for watermarking of 3D Images.

In [7], two novel methods suitable for blind 3D mesh object watermarking applications are proposed. The first method is robust against 3D rotation, translation, and uniform scaling. The second one is robust against both geometric and mesh simplification attacks. In the first method, a set of vertices that correspond to specific angles /spl theta/ is used for watermark embedding. In the second method, the samples of the watermark sequence are embedded in a set of vertices that correspond to a range of angles in the /spl theta/ domain in order to achieve robustness against mesh simplifications. Prior to watermark embedding and detection, the object is rotated and translated so that its center of mass and its principal component coincide with the origin and the $\mathrm{z}$-axis of the Cartesian coordinate system. This geometrical transformation ensures watermark robustness to translation and rotation. Robustness to uniform scaling is 


\section{International Advanced Research Journal in Science, Engineering and Technology}

Vol. 6, Issue 3, March 2019

achieved by restricting the vertex deformations to occur only along the $r$ coordinate of the corresponding spherical coordinate system. A reversible data hiding method to authenticate $3 \mathrm{D}$ meshes by modulating the distances from the mesh faces to the mesh centroid to embed a fragile watermark is proposed in [8].

It keeps the modulation information in the watermarked mesh so that the reversibility of the embedding process is achieved. Since the embedded watermark is sensitive to geometrical and topological processing, unauthorized modifications on the watermarked mesh can be therefore detected by retrieving and comparing the embedded watermark with the original one. Furthermore, as long as the watermarked mesh is intact, the original mesh can be recovered using some priori knowledge. A digital watermarking technique for 3D design drawing using Line and 3DF ACE based on vertex in CAD system to prevent infringement of copyright from unlawfulness reproductions and distribution is proposed in [9].An original hierarchical watermarking scheme for three-dimensional triangular meshes is proposed in [10]. A geometrically robust watermark and a high-capacity watermark are inserted in different resolution levels of the wavelet decomposition of a semi-regular mesh by modifying the norms of wavelet coefficients. Both watermarks are blind and invariant to similarity transformations. The robustness of the first watermark is achieved by synchronizing and quantizing watermark primitives according to edges lengths of the coarsest level, which are quite insensible to geometrical attacks. The high capacity of the second watermark is obtained by considering the permutation of the norms of a group of wavelet coefficients.

[11] proposes a new semi-fragile watermarking algorithm for the authentication of 3D models based on integral invariants. A watermark image is embedded by modifying the integral invariants of some of the vertices. In order to modify the integral invariants, the positions of a vertex and its neighbors are shifted. To extract the watermark, all the vertices are tested for the embedded information, and this information is combined to recover the watermark image. The number of parts of the watermark image that can be recovered will determine the authentication decision.Idea in [12] is to adapt watermark strength to local surface roughness based on the knowledge that human eyes are less sensitive to changes on a rougher surface patch than those on a smoother surface. In order to quantify human sensitivity to surface roughness of polygonal meshes, we conducted a rigorous psychovisual experiment to obtain human watermark detection thresholds as a function of surface roughness. The results can be used to adaptively select watermark strength according to local surface roughness during the watermark embedding process. The depth-image-based rendering (DIBR) 3D image is one of the image-based 3D data which consists of the center image and the depth image generated by the content provider[13]. The left-eye image and the right-eye image are rendered from the center image and the depth image at the content consumer side. The blind watermarking for DIBR 3D image is rarely studied in the literature. A novel blind multiple watermarking scheme is proposed to deal with the content protection problem of DIBR 3D images[13].

A robust watermarking scheme for DIBR 3D images by quantization on dual-tree complex wavelet transform (DTCWT) coefficients with consideration of imperceptibility is given in [14]. Two characteristics of DT-CWT are employed: approximate shift invariance and directional selectivity. They selected certain coefficient sub-blocks and group the coefficient rows based on the properties of DIBR. On the extraction side, the threshold is carefully chosen with a low false positive rate. The simulation results show that the embedded watermark is stably extracted from the center view and the synthesized left and right views. In addition, even if the synthesized left and right views are distorted by general attacks, the watermark is successfully extracted. A new 3D surface preservation function metric is defined consisting of the distance of a vertex displaced by watermarking to the original surface, to the watermarked object surface as well as the actual vertex displacement[15].The proposed method[15] is statistical, blind, and robust. Minimal surface distortion according to the proposed function metric is enforced during the statistical watermark embedding stage using Levenberg-Marquardt optimization method.

In [16] , a digital watermarking method for depth-image-based rendered 3D video is proposed. In this method, the watermark is embedded in both of the chrominance channels of a YUV representation of the center view using the dualtree complex wavelet transform. Then, the left and right views are generated from the watermarked center view and depth map using a depth-image based rendering technique. Finally, the watermark can be extracted from the center, left, and right views in a blind fashion without using the original unwatermarked center, left, or right views. This watermark is robust to geometric distortions, such as upscaling, rotation and cropping, downscaling to an arbitrary resolution, and the most common video distortions, including lossy compression and additive noise. A 3D model watermarking method robust to geometric attacks is proposed in [17]. The vertices of the model are classified into three groups. The vertices of the low-resolution group are used to establish an invariant space in which to resist geometric attacks. In the mediumresolution group, appropriate vertices in which to embed the watermarking information are selected. The selection of vertices that contain information about the watermark is based on the area of the local set of the vertex, the curvature of which determines the embedding strength of the watermark. The vertices of the high-resolution group are reserved for resisting simplification and smoothing attacks. The choice of the embedding position and the embedding strength can 


\section{International Advanced Research Journal in Science, Engineering and Technology}

Vol. 6, Issue 3, March 2019

provide a suitable trade-off between good transparency and maximum robustness of the proposed method. A new blind watermarking scheme for 3-D point cloud models by using feature vertices to locate the embedded watermark is proposed in [18].

The vertices with larger mean curvature are considered to be feature vertices carrying watermarking information; nonfeature vertices are used to build a new coordinate system in which the 3-D point cloud model is classified into bins containing several longitudes. Each bit of the watermarking information is repeatedly embedded into a bin by altering azimuth angles of feature vertices lying in the bin. The non-feature vertices are not exploited to embed the watermarking information, which enables the built coordinate system to avoid the impacts of the embedding of watermark. The choice of the embedding position and redundant embedding provide an optimal balance between imperceptibility and robustness of watermark. The method in [19] maps decimals of the vertex coordinates into integers first, so that a bit-stream encryption technique can be executed. With a data-hiding key, several least-significant bits are operated to embed data. By using the encryption key, a receiver can roughly reconstruct the content of the mesh. According to the data-hiding key, with the aid of spatial correlation in natural mesh models, the embedded data can be successfully extracted and the original mesh can be perfectly recovered.

[20] presents a review of the digital video watermarking techniques in which their applications, challenges, and important properties are discussed, and categorizes them based on the domain in which they embed the watermark. It then provides an overview of a few emerging innovative solutions using watermarks. Protecting a 3D video by watermarking is an emerging area of research. The relevant 3D video watermarking techniques in the literature are classified based on the image-based representations of a 3D video in stereoscopic, depth-image-based rendering, and multi-view video watermarking.

In [21], a histogram shape based watermarking algorithm is proposed to protect the DIBR 3D images. To make the watermarking method robust to common attacks, a pixel mean value based pixel groups selection method is presented to select several suitable pixel groups for watermark embedding. To solve the problem that the dividing of pixel groups can affect the performance of watermark extraction, the width of pixel group is determined by the maximum difference of pixel mean value between the original and attacked images. By this way, the watermark can be extracted with lower bit error rate(BER) from the attacked watermarked image. Also This method has good robustness to the adjusting of baseline distance and depth image blurred. Depth-Image-Based Rendering (DIBR) has been recently recognized as an effective method to synthesize 3D content due to its low bandwidth requirement and depth adjustment capability[22]. However, as important it is to generate the DIBR views, it is also important to preserve the copyright of DIBR's views when subjected to illegal manipulation and distribution. A blind, imperceptible, and robust 3D DIBR digital image watermarking algorithm based on cascading two mathematical transforms, the Discrete Wavelet Transform (DWT) and the Singular Value Decomposition (SVD) are proposed in [22].

The two transforms provide different, but complementary, levels of robustness against watermarking attacks. Moreover, a watermark is hidden using a chaos-based embedding procedure, and extracted blindly at the receiver side, not only from the center image, but also from the synthesized left-eye and right-eye views. Imperceptibility and robustness of the proposed algorithm are demonstrated using a benchmark set of monoscopic images and their corresponding per-pixel depth map images. The paper[23] presents a 3D watermark based medical image watermarking scheme. In this paper, a $3 \mathrm{D}$ watermark object is first decomposed into 2D elemental image array (EIA) by a lenslet array, and then the 2D elemental image array data is embedded into the host image. The watermark extraction process is an inverse process of embedding. The extracted EIA through the computational integral imaging reconstruction (CIIR) technique, the 3D watermark can be reconstructed. Because the EIA is composed of a number of elemental images possesses their own perspectives of a 3D watermark object. Even though the embedded watermark data badly damaged, the 3D virtual watermark can be successfully reconstructed. Furthermore, using CAT with various rule number parameters, it is possible to get many channels for embedding. So our method can recover the weak point having only one transform plane in traditional watermarking methods. The research idea of 3D geological body digital watermarking algorithm based on point cloud model is proposed in [24].

According to the spatial characteristics of the point cloud model of geological body, the spatial characteristic variables of the point cloud model are obtained by using the spatial characteristic analysis algorithm. The spatial domain information represented by the spatial characteristic variables is transformed into the frequency domain information, and then the frequency domain information is analyzed by means of mathematical statistics to extract the digital watermarking information of the whole geological body point cloud model. In the proposed scheme of [25] master shares are first generated by extracting features from temporally informative representative images (TIRIs) of both the 2D video frames and depth maps. Then, ownership shares, which denote the relationships between copyright 


\section{International Advanced Research Journal in Science, Engineering and Technology}

Vol. 6, Issue 3, March 2019

information and master shares, are generated based on the Visual Secret Sharing (VSS) scheme and stored for copyright identification. Finally, copyright ownerships are identified by stacking master shares and ownership shares.

\section{CONCLUSIONS}

As the 3D display market has been grown rapidly, the demand for 3D content has also increased in parallel. In fact, various recent movies have been produced in 3D form, and audiences have shown great interest in immersive experiences through the 3D content. Those 3D contents are represented by two methods. One is called stereo image recording (SIR), which is to store the left and right views, which are simultaneously captured for the same scene from different camera locations. This method offers a high quality viewing environment to viewers because there are two capture devices that act like human eyes. Despite such an advantage, the method has several drawbacks. It is very difficult and costly to set two cameras with the same contrast, brightness, and height. Also, the initial depth condition is fixed. That is, viewers are not able to adjust the depth condition they prefer. Large storage or transmission bandwidth due to the double color images for one scene is a critical issue as well. The other 3D representation method is depthimage-based rendering (DIBR) [26], [27]. Both the left and right views are generated by a monoscopic center view and associated per-pixel depth information using the DIBR technique. Contrary to the SIR, it provides several advantages. Firstly, viewers can easily adjust the depth degree by modifying the depth image in the DIBR system. In other words, the DIBR system provides individualized depth configuration. Secondly, it has a lower transmission bit rate per scene [28] because the depth image consists of gray level pixels and many smooth areas that lead to a higher compression ratio. Lastly, the DIBR system has backwards-compatibility to 2D digital TVs because the center image itself can be utilized as 2D content. Due to the numerous advantages of the DIBR system, many studies have been conducted on DIBR and its applications. In particular, research on watermarking for copyright protection of DIBR content has recently received attention due to the rapid growth of the 3D content market. A copy- right protection technique should consider situations not only in which both virtual left and right views are illegally distributed as 3D content but also in which each single view, including the original center view, is leaked to the public without permission. So far, numerous digital watermarking schemes for 2D and SIR images have been presented [29]-[32]. In DIBR 3D images, how- ever, pixels of a watermarked center image are partially moved horizontally to different distances due to the DIBR process. Furthermore, the corresponding depth images can be modified for a certain purpose after the watermark embedding. For those reasons, conventional watermarking schemes for 2D and stereo images cannot be directly applied to the DIBR 3D images.

The applications involving 3D data are quickly increasing in number and many efforts to obtain 3D models by direct acquisition or modeling have been deployed. Additionally, the computer graphics power reached by personal computers and Internet have incremented the diffusion of this new media. For these reasons, the interest in developing techniques to provide IPR protection of 3D models is increasing. Watermarking technology has been successfully used for this purpose for other kinds of media such as images and video, but, a lot of work is again necessary to reach robustness and industrial applicability for 3D watermarking algorithms.

\section{REFERENCES}

[1]. https://en.wikipedia.org/wiki/Digital_watermarking

[2]. Smita Jagdishprasad Jaipuria ,Watermarking for Depth Map Based 3D images using wavelet transform ,2014 International Conference on Communication and Signal Processing, IEEE Conferences.

[3]. Shinichi Nakazawa ; Sho Kasahara ; Shigeo Takahashi, A Visually Enhanced Approach to Watermarking 3D Models, 2010 Sixth International Conference on Intelligent Information Hiding and Multimedia Signal Processing, IEEE Conferences.

[4]. J.-S. Pan, H.-C. Huang, L. C. Jain, and W.-C. Fang, Eds., Intelligent Multimedia Data Hiding: New Directions. Springer,2007.

[5]. Salma Elsherif ; Ghadir Mostafa ; Sara Farrag ; Wassim Alexan ,Secure Message Embedding in 3D Images ,2019 International Conference on Innovative Trends in Computer Engineering (ITCE), IEEE Conferences

[6]. https://en.wikipedia.org/wiki/Wavefront_.obj_file

[7]. S. Zafeiriou ; A. Tefas ; I. Pitas ,Blind robust watermarking schemes for copyright protection of 3D mesh objects ,IEEE Transactions on Visualization and Computer Graphics IEEE Journals \& Magazines 2005.

[8]. Hao-Tian Wu ; Yiu-ming Cheung ,A reversible data hiding approach to mesh authentication ,The 2005 IEEE/WIC/ACM International Conference on Web Intelligence (WI'05), IEEE Conferences.

[9]. Ho-Kyun Park ; Suk-Hwan Lee ; Ki-Ryong Kwon ,Blind Watermarking for Copyright Protection of 3D CAD Drawing ,2006 8th International Conference Advanced Communication Technology ,IEEE Conferences

[10]. Kai Wang ; Guillaume Lavoue ; Florence Denis ; Atilla Baskurt ,Hierarchical Blind Watermarking of 3D Triangular Meshes ,2007 IEEE International Conference on Multimedia and Expo, IEEE Conferences

[11]. Yu-Ping Wang ; Shi-Min Hu ,A New Watermarking Method for 3D Models Based on Integral Invariants, IEEE Transactions on Visualization and Computer Graphics ,IEEE Journals \& Magazines

[12]. Kwangtaek Kim ; Mauro Barni ; Hong Z. Tan ,Roughness-Adaptive 3-D Watermarking Based on Masking Effect of Surface Roughness, IEEE Transactions on Information Forensics and Security ,Volume: 5 , Issue: 4 ,IEEE Journals \& Magazines 2010.

[13]. Yu-Hsun Lin ; Ja-Ling Wu, A Digital Blind Watermarking for Depth-Image-Based Rendering 3D Images, IEEE Transactions on Broadcasting ,Volume: 57 , Issue: 2 ,IEEE Journals \& Magazines ,2011.

[14]. Ji-Won Lee ; Tae-Woo Oh ; Heung-Kyu Lee , Robust DT-CWT Watermarking for DIBR 3D Images, IEEE Transactions on Broadcasting, Volume: 58 , Issue: 4 ,IEEE Journals \& Magazines,2012. 


\title{
International Advanced Research Journal in Science, Engineering and Technology
}

\author{
Vol. 6, Issue 3, March 2019
}

[15]. Adrian G. Bors ; Ming Luo ,Optimized 3D Watermarking for Minimal Surface Distortion, IEEE Transactions on Image Processing, Volume: 22, Issue: 5 ,IEEE Journals \& Magazines 2013.

[16]. Md. Asikuzzaman ; Md. Jahangir Alam; Andrew J. Lambert, Robust DT CWT-Based DIBR 3D Video Watermarking Using Chrominance Embedding ,IEEE Transactions on Multimedia ,Volume: 18, Issue: 9, IEEE Journals \& Magazines 2016.

[17]. Yinghui Wang ; Jing Liu ; Yajie Yang ; Douli Ma ; Ruijiao Liu ,3D model watermarking algorithm robust to geometric attacks ,IET Image Processing ,Volume: 11 , Issue: 10 ,IET Journals \& Magazines 2017.

[18]. Jing Liu ; Yajie Yang ; Douli Ma ; Yinghui Wang ; Zhigeng Pan ,A Watermarking Method for 3D Models Based on Feature Vertex Localization, Volume: 6 ,IEEE Journals \& Magazines 2018

[19]. Ruiqi Jiang ; Hang Zhou ; Weiming Zhang ; Nenghai Yu ,Reversible Data Hiding in Encrypted Three-Dimensional Mesh Models ,IEEE Transactions on Multimedia ,Volume: 20,Issue: 1 ,IEEE Journals \& Magazines 2018.

[20]. Md. Asikuzzaman ; Mark R. Pickering, An Overview of Digital Video Watermarking ,IEEE Transactions on Circuits and Systems for Video Technology ,Volume: 28 , Issue: 9, IEEE Journals \& Magazines 2018.

[21]. C. Cui, X-M. Niu, A Robust DIBR 3D Image Watermarking Algorithm Based on Histogram Shape, Measurement (2016), doi: http://dx.doi.org/10.1016/j.measurement.2016.05.079

[22]. A. Al-Haj, M.E. Farfoura, A. Mohammad, Transform-Based Watermarking of 3D Depth-Image- based-Rendering Images, Measurement (2016), doi:http://dx.doi.org/10.1016/j.measurement.2016.10.016

[23]. Xiao WeiLi, SeokTaeKim ,Optical3Dwatermarkbaseddigitalimagewatermarking for telemedicine, Volume 51, Issue 12, December 2013,Optics andLasersinEngineering51(2013),1310-1320, www.elsevier.com/locate/optlaseng.

[24]. Xialin Zhang, Zaiquan Shen, Copyright protection method for 3D model of geological body based on digital watermarking technology, Journal of Visual Communication and Image Representation, Volume 59, February 2019.

[25]. Xiyao Liu, Rongchang Zhao, Fangfang Li, Shenghui Liao, Beiji Zou ,Novel robust zero-watermarking scheme for digital rights management of 3D videos Signal Processing:Image Communication, Volume 54, May 2017.

[26]. C. Fehn, P. Kauff, M. Op de Beeck, F. Ernst, W. Ijsselsteijn, M. Polle- feys, L. Vangool, E. Ofek, and I. Sexton, "An evolutionary and optimised approach, on 3D-TV," in IBC 2002, Int. Broadcast Convention, Amsterdam, Netherlands, Sept. 2002

[27]. C. Fehn, "Depth-image-based rendering (DIBR), compression and transmission for a new approach on 3D-TV," in Proc. SPIE Conf.Stereoscopic Displays and Virtual Reality Systems XI, 2004, vol. 5291,pp. 93-104.

[28]. Y. C. Fan and T. C. Chi, "The novel non-hole-filling approach of depth image based rendering," in 3DTV, 2008, pp. 325-328.

[29]. D. Zheng, S. Wang, and J. Zhao, "RST invariant image watermarking algorithm with mathematical modeling and analysis of the watermarking process," in IEEE Trans. on Image Processing, May 2009, vol. 18, no. 5.

[30]. C. Deng, X. Gao, X. Li, and D. Tao, “A local Tchebichef moments- based robust image watermarking,” in Signal Processing, Aug. 2009 , vol. 89, no. 8, pp. $1531-1539$.

[31]. D. C. Hwang, K. H. Bae, and E. S. Kim, "Stereo image watermarking scheme based on discrete wavelet transform and adaptive disparity estimation," Mathematics of Data/Image Coding, Compression, and Encryption VI, With Applications, vol. 5208, no. 1, pp. 196-205, 2004.

[32]. A. Chammem, M. Mitrea, and F. Prłteux, "DWT-based stereoscopic image watermarking," in Proc. SPIE 7863, 2011, p. 786326. 